CERN LIBRARIES, GENEVA

\author{
F. Halzen \\ CERN, Geneva \\ J. Mandula *)
}

California Institute of Technology, Pasadena

J. Weyers

California Institute of Techrology, Pasadena and

CERN, Geneva

$$
\text { G. Zweig } \left.{ }^{*} *^{* *}\right)
$$

California Institute of Technology, Pasadena

\title{
ABSTRACT
}

Many relations which sensitively express the suppression of exotic exchanged quantum numbers are derived and compared with the available data.

*) Work supported in part by the U.S. Atomic Energy Commission. Prepared under contract AT(11-1)-68 for the San Francisco Operations Office, U.S. Atomic Energy Commission.

**) Alfred P. Sloan Foundation Fellow. 
Most phenomenological analyses of high energy scattering describe reactions in terms of their crossed channel exchanges. It is therefore desirable to organize scattering data so that the contributions of each set of crossed channel quantum numbers are isolated. We know from examination of reactions where only exotic quantum numbers are exchanged that exchanges with these quantum numbers are strongly suppressed. Their size is not known quantitatively, however. A sensitive way to measure these exotic exchanges is to interfere them with the dominant non-exotic exchanges. We catalogue here all relations following from the absence of exotic exchanges which have the property that their violation is proportional to the interference between an exotic and a non-exotic exchange, rather than to the square of an exotic exchange.

Many of these relations have been described previously and are included here only for completeness ${ }^{1}$ ). The compendium, which is in Table I, contains two classes of relations. Class I relations depend only on the absence of exotic exchange while Class II relations depend in addition on exact $\mathrm{SU}_{3}$ symmetry. We have omitted relations between reactions with different crossed channel isotopic spins, since they, of course, are expected to have different energy dependences.

An instructive way to present the data correlated by these relations would be to calculate their percentage of violation as a function of energy and angle for each reaction. A "contour map", with percentage violation plotted as height on the s-t plane, would clearly show the limits of the purely non-exotic exchange approximation, and might give clues about what are the important dynamical effects in various parts of the physical region.

At present there are data bearing on some of these relations. Unfortunately, the data is not precise enough to find the contours of constant fractional violation. This is especially true at high energies, where the relations roughly hold. So rather than constmcting contour maps of the violations of these relations at this time, we will instead present for each relation, at each value of the momentum transfer for which there is data, a plot of the right and left-hand sides, as a function of energy, on the same graph, along with a plot of the fractional differences between the right and left-hand sides. The data ${ }^{2)}$ are shown in Figs. 1-15.

We would like to make several remarks about possible uses of the se relations and about expectations based on currently popular models.

a) It would be especially interesting to compare reactions that involve currents (photoproduction, Compton scattering, and inelastic electron and neutrino scattering at various fixed $\mathrm{q}^{2}$ ) to purely hadronic reactions. If the "contours of constant percentage violation" have the same shape in both kinds of reations, one would be fairly confident that the same dynamical approximations could be applied to both. But if the contour maps were qualitatively different, then different dynamical descriptions of each type of reaction would have to be sought. 
b) If the Class I relations are very accurately satisfied in some region of energy and angle, then one could assume that in that part of the physical region the approximation of no exotic exchange is very accurate. In the same part of the physical region the violation of Class II relations will then constitute a measurement of the violation of $\mathrm{SU}_{3}$ symmetry. Examination of reactions involving currents may allow one in addition to examine $\mathrm{SU}_{3}$ symmetry breaking as a function of the mass of the current.

c) At low energies where there are prominent resonances with definite direct channel quantum numbers, the se relations must be strongly violated. It is possible that the relations correlating baryon number two reactions are more accurate at low energies since there are no strong resonances in this channel ${ }^{3)}$.

The remaining remarks are made within the Regge phenomenological framework.

d) Regge poles and their associated diffractive cuts have the same internal quantum numbers and so these cuts do not lead to any violation of the "no-exotics" relations.

e) Regge asymptotic behaviour asserts that lines of fixed $t$ on the $s-t$ plane will cross contours of constant percentage violation. For fixed $t$, the relations will become exact as $s$ goes to infinity. If the potential theoretic intuition that the magnitude of the crossed cosine determines the accuracy of the Regge asymptotic formula is correct, then the contours of constant violation will asymptote to rays of fixed $t / s$, at least for small $t / s$.

One of the authors (J.W.) would like to thank Professor Murray Gell-Mann for his hospitality at the California Institute of Technology. 
The relations given are between the cross-sections of the indicated processes. Class I reactions depend only on the absence of crossed channel exotic contributions, while class II reactions depend on exact $\mathrm{SU}_{3}$ symmetry as well. Except for the two relations involving four cross-sections, they are all the squares of relations between amplitudes and so the polarizations of the particles in related reactions should be equal. The particle names denote only the internal quantum numbers of the indicated particles so that $\pi^{+}$, for example, may stand for $\pi^{+}(140), \rho^{+}(765), A_{1}^{+}(1070)$, $\mathrm{A}_{2}^{+}(1305)$.... An octet of mesons is denoted by $\mathbb{M}$, an octet of baryons by $B$, and a decuplet of baryons by $\Delta \cdot \boldsymbol{\Sigma}^{*}$ and $\Xi^{*}$ denote members of a decuplet. The relations enclosed in \{\} are the squares of relations which could be obtained by isotopic rotations from other amplitude relations whose squares appear in the Table. However, because the relative phases of the isospin eigenamplitudes are unknown, these cross-section relations are in fact independent. The Cabibbo angle is denoted by $\theta$.

\section{$M B-M^{\prime} B^{\prime}$}

$\pi^{+} \mathrm{p} \rightarrow \mathrm{K}^{+} \Sigma^{+}=2\left(\pi^{-} \mathrm{p} \rightarrow \mathrm{K}^{0} \Sigma^{\circ}\right)$

$K^{-} p \rightarrow \pi^{-} \Sigma^{+}=2\left(K^{-} n \rightarrow \pi^{-} \Sigma^{\circ}\right)$

$\left(K^{-} p-\vec{K}^{0} n\right)+\left(K^{+} n-K^{0} p\right)=\left(\pi^{-} p-\pi^{0} n\right)+3\left(\pi^{-} p-\eta n\right)$

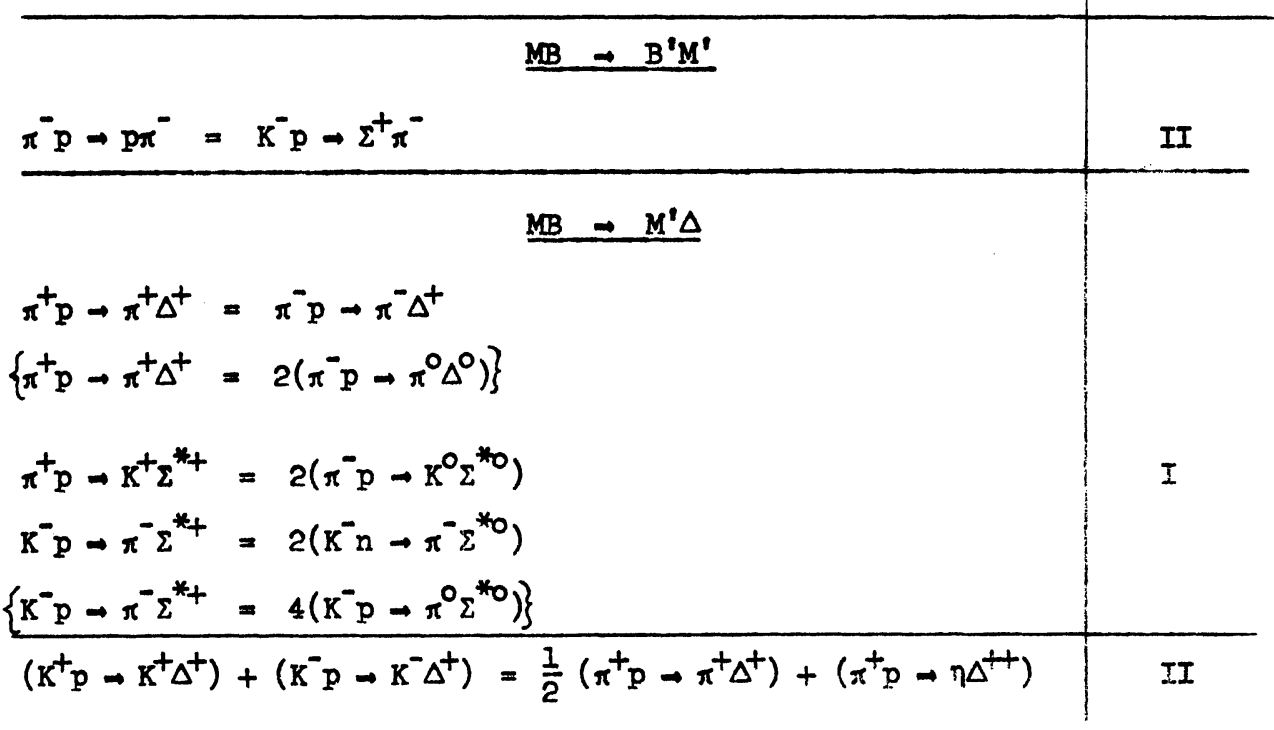


TABLE I (cont.)

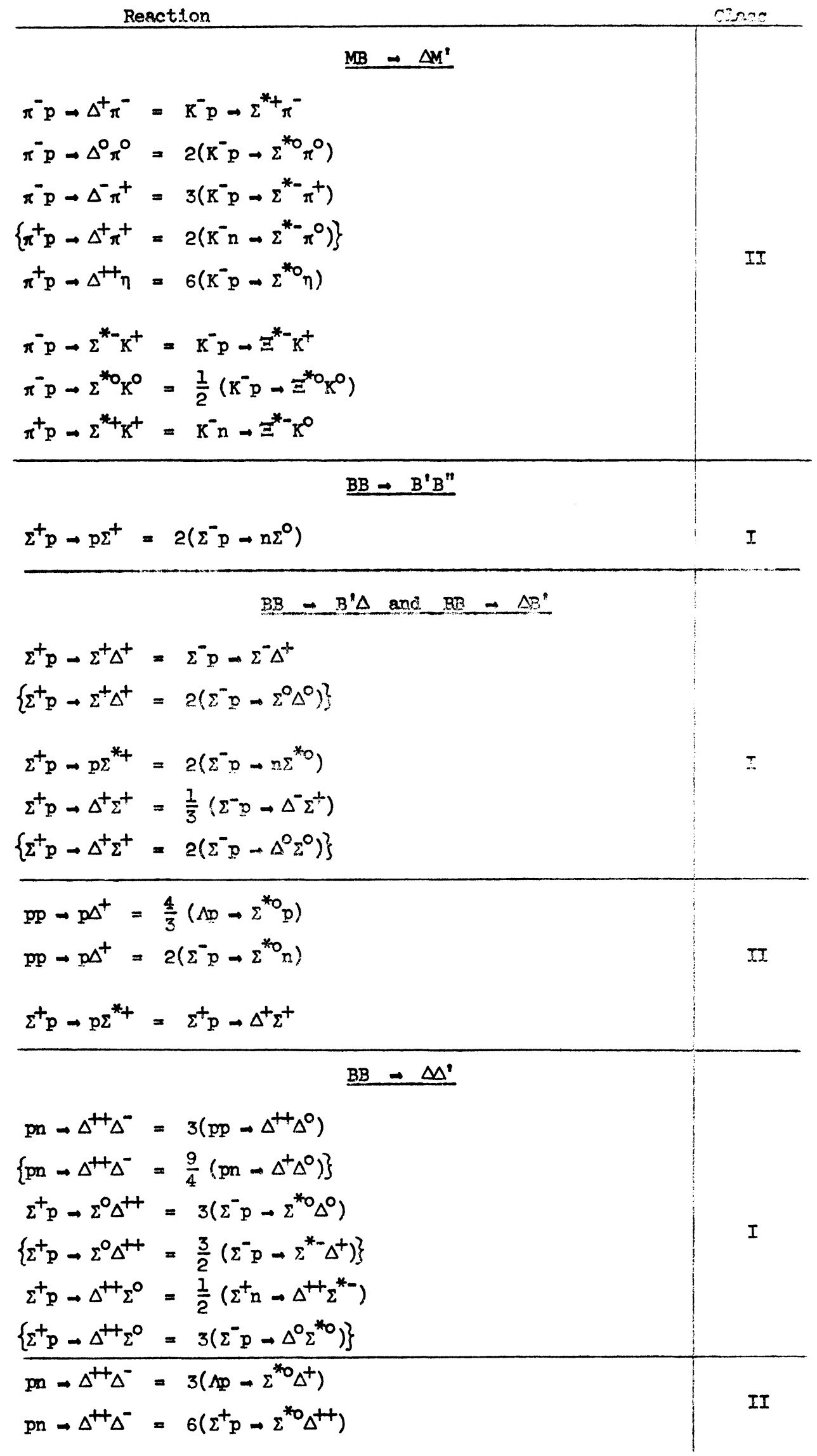


TARIE I (cont.)

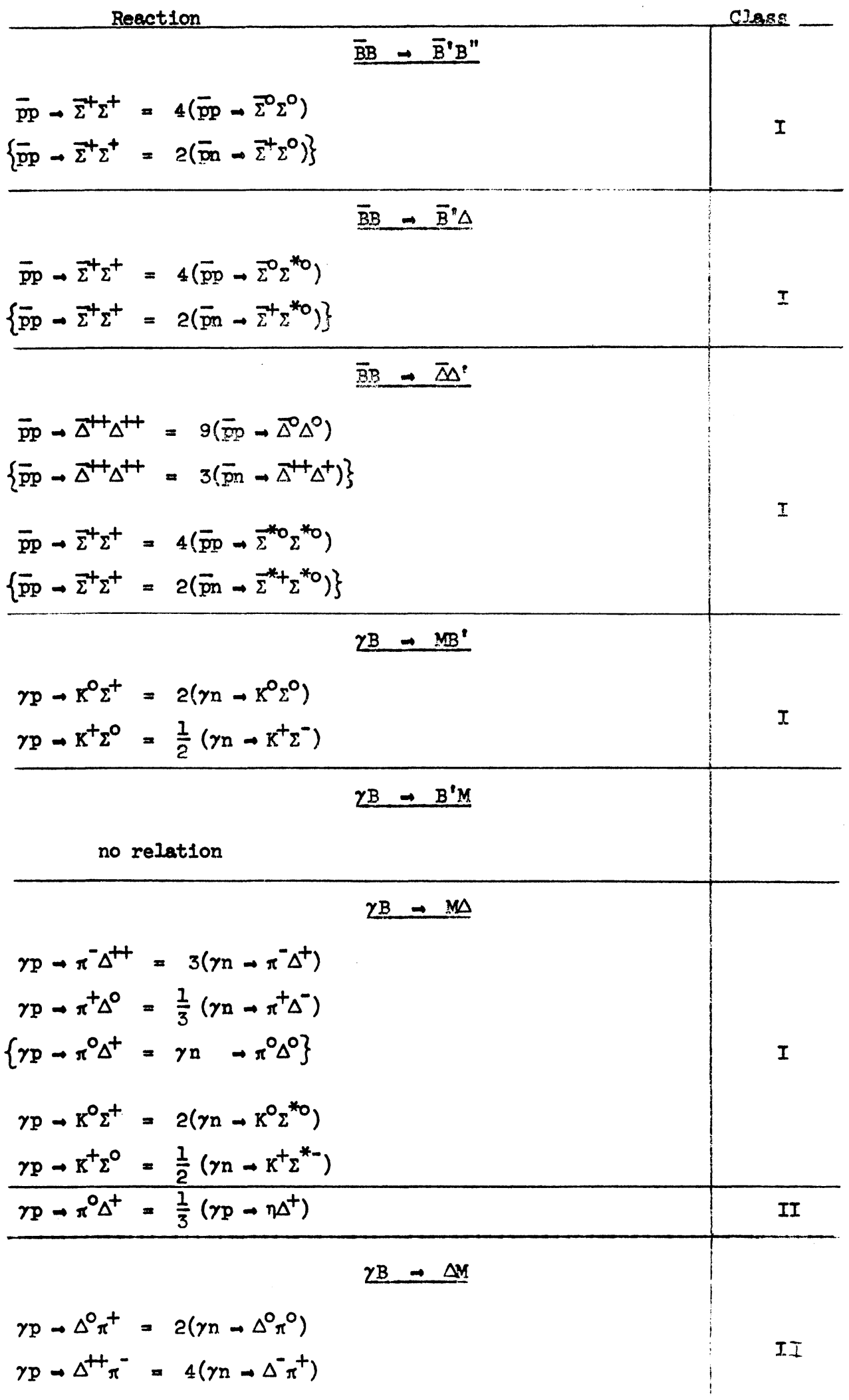


- 9 -

NABIEI (cont.)

Reaction

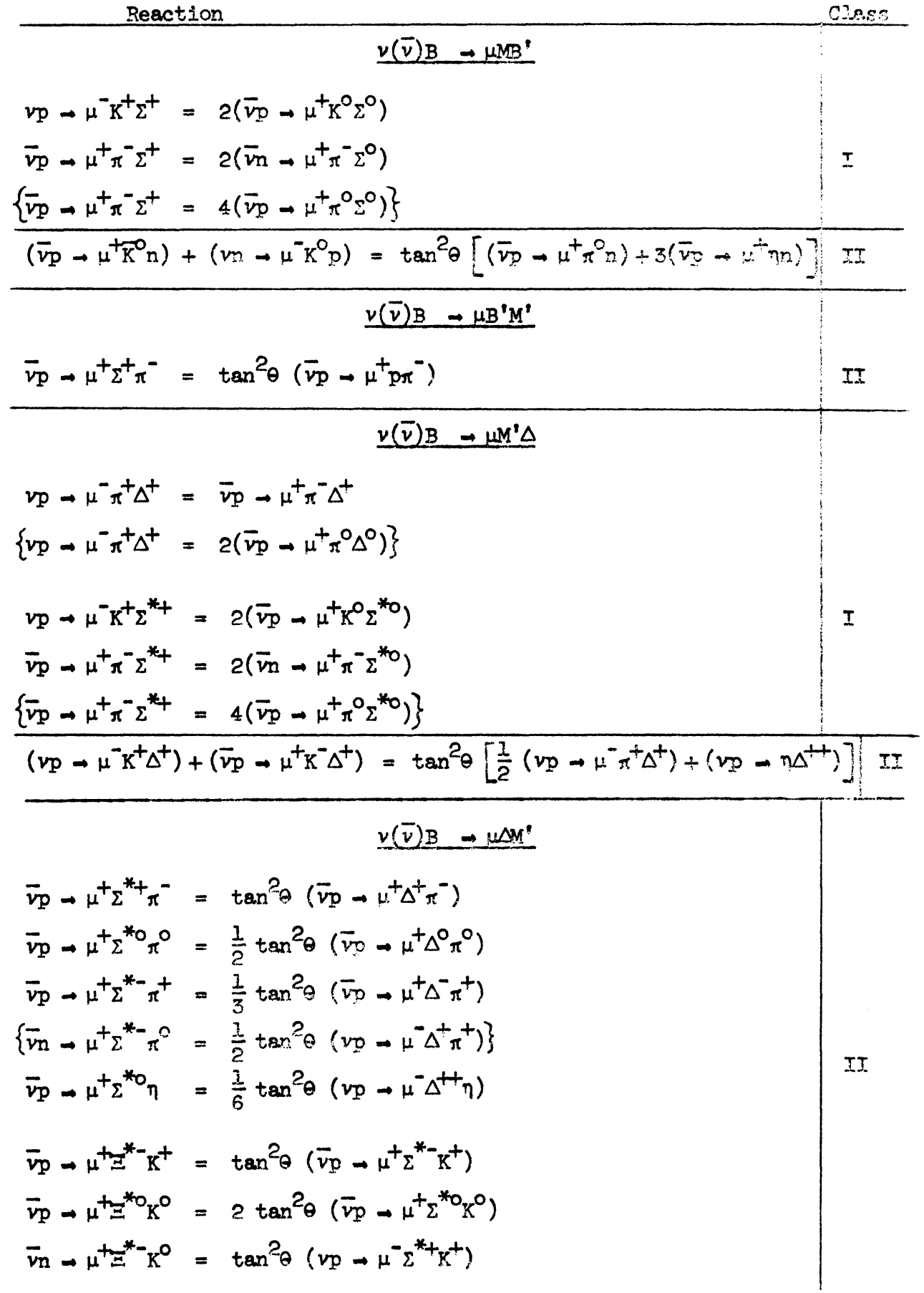


REFERENCES

1) See V. Barger and D. Cline, Phys. Rev. 156, 1522 (1967);

A.M. Boyarski, R. Diebold, S.D. Ecklund, G.E. Fisher, Y. Murata, B. Richter and M. Sands, Phys. Rev. Letters 25, 695 (1970);

M. Abramovich, H. Blumenfeld, V. Chaloupka, S.U. Chung, J. Diaz, I. Montanet, J. Pernegr, S. Rencroft, J. Rubio and B. Sadoulet, CERN Preprint PHYS. 70-6I, to be published in Nucl. Phys. B.

2) Data references:

$$
\begin{aligned}
& \mathrm{K}^{-} \mathrm{p} \rightarrow \pi^{-} \Sigma^{+} \quad \text { at } 3 \mathrm{GeV} / \mathrm{c} \quad \text { J. Badier et al., Report CEA-R-3037 (1966); } \\
& \text { at } 4.07 \mathrm{GeV} / \mathrm{c} \text { J.S. Loos, V.E. Kruse and E.I. Goldwasser, } \\
& \text { Phys. Rev. 173, } 1330 \text { (1968); } \\
& \mathrm{K}^{-} \mathrm{n} \rightarrow \pi^{-} \Sigma^{\circ} \quad \text { at } 3 \mathrm{GeV} / \mathrm{c} \quad \text { SABRE Collaboration, Nucl. Phys. B9, } \\
& 493 \text { (1969); } \\
& \pi^{-} p \rightarrow K^{0} \Sigma^{0} \quad \text { at } 2.9-3.3 \mathrm{GeV} / \mathrm{c} \\
& \begin{array}{l}
\text { at } 4.5 \mathrm{GeV} / \mathrm{c} \\
\text { at } 2.9-3.3 \mathrm{GeV} / 0
\end{array} \\
& \text { at } 3.9 \mathrm{GeV} / \mathrm{c} \\
& \pi^{+} \mathrm{p} \rightarrow \mathrm{K}^{+} \boldsymbol{\Sigma}^{+} \quad \text { at } 3,3.25 \text { and } \\
& \pi^{-p} \rightarrow \pi^{\circ} \mathrm{n} \quad \text { at } 3,4.83 \text { and } \\
& 13.3 \mathrm{GeV} / \mathrm{c} \\
& \text { W.I. Yen et al., Phys. Rev. 188, } 2011 \text { (1969); } \\
& \text { O.I. Dahl et aI., Phys. Rev. 163, } \\
& \text { 1430 (1967); } \\
& \text { M. Abramovich et al., CERN/DPhII/ } \\
& \text { Phys. 70-61; } \\
& \text { S.M. Pruss et al., Phys. Rev. Letters } \\
& \text { 23, } 189 \text { (1969); } \\
& \text { P. Sonderegger et al., Phys. Letters } \\
& \text { 20, } 75 \text { (1900); } \\
& \begin{array}{l}
\pi^{-} \mathrm{p} \rightarrow \eta^{\mathrm{n}} \\
\mathrm{K}^{-} \mathrm{p} \rightarrow \overline{\mathrm{K}}^{\bar{b}} \mathrm{n}
\end{array} \\
& \mathrm{K}^{+} \mathrm{n} \rightarrow \mathrm{K}^{\mathrm{O}} \mathrm{p} \\
& \text { at } 2.91,5.9 \text { and } \\
& 13.3 \mathrm{GeV} / \mathrm{c} \\
& \text { at } 3 \mathrm{GeV} / \mathrm{c} \\
& \text { at } 5 \text { and } \\
& \text { 12. } 3 \mathrm{GeV} / \mathrm{c} \\
& \text { at } 3 \mathrm{GeV} / \mathrm{c} \\
& \text { at } 5.5 \mathrm{GeV} / \mathrm{c} \\
& \text { at } 12 \mathrm{GeV} / \mathrm{c} \\
& \pi^{-} p \rightarrow \pi^{-} \Delta^{+} \\
& \text {at } 8 \mathrm{GeV} / \mathrm{c} \\
& \pi^{+} p \rightarrow \pi^{\circ} \Delta^{++} \quad \text { at } 8 \mathrm{GeV} / \mathrm{c} \\
& \pi^{+} \mathrm{p} \rightarrow \pi^{\circ} \Delta^{++} \quad \text { at } 5 \mathrm{GeV} / \mathrm{c} \\
& \pi^{+}{ }_{p} \rightarrow \eta \Delta^{++} \quad \begin{array}{l}
\text { at } 3-4 \mathrm{GeV} / \mathrm{c} \\
\text { at } 3.5 \mathrm{GeV} / \mathrm{c}
\end{array} \\
& \text { at } 5 \mathrm{GeV} / \mathrm{c} \\
& \text { at } 8 \mathrm{GeV} / \mathrm{c} \\
& \mathrm{K}^{-} \mathrm{n} \rightarrow \overline{\mathrm{K}^{\mathrm{O}}} \Delta^{-} \quad \text { at } 3 \mathrm{GeV} / \mathrm{c} \text { and } \\
& \text { 0. Guisan et al., Phys. Letters 18, } \\
& \text { J. Badier et al., Report CEA-R-3037 (1966); } \\
& \text { P. Astbury et al., Phys. Letters 23, } \\
& 396 \text { (1966); } \\
& \text { Y. Goldschmidt-Clermont et al., Phys. } \\
& \text { Letters 27B, } 602 \text { (1968); } \\
& \begin{array}{l}
\text { D. Cline et al., Nucl. Phys. B22, } \\
\text { 247 (1970); }
\end{array}
\end{aligned}
$$




$$
\begin{array}{ll}
\mathrm{K}^{-} \mathrm{p} \rightarrow \mathrm{K}^{-} \Delta^{+} & \text {at } 10 \mathrm{GeV} / \mathrm{c} \\
\mathrm{K}^{+} \mathrm{p} \rightarrow \mathrm{K}^{\circ} \Delta^{++} & \begin{array}{l}
\text { at } 3.5,5 \text { and } \\
8.25 \mathrm{GeV} / \mathrm{c}
\end{array} \\
\pi^{+} \mathrm{p} \rightarrow \rho(\omega) \Delta^{++} & \text {at } 3.7 \mathrm{GeV} / \mathrm{c} \\
\mathrm{K}^{-} \mathrm{n} \rightarrow \overline{\mathrm{K}}^{-*} \Delta^{-} & \text {at } 5 \mathrm{GeV} / \mathrm{c} \\
\mathrm{K}^{+} \mathrm{p} \rightarrow \mathrm{K}^{\mathrm{O}^{*}} \Delta^{++} & \text {at } 3 \mathrm{GeV} / \mathrm{c} \\
& \text { at } 5.5 \mathrm{GeV} / \mathrm{c} \\
& \text { at } 3.5 \mathrm{GeV} / \mathrm{c}
\end{array}
$$

Photoproduction data
Aachen-Berlin-CERN-Iondon-Vienna Collaboration, Nucl. Phys. B7, IIl (1968);

CERN-Bruxelles Collaboration, Vienna International Conference on High Energy Physics (paper no. 445); (1968);

G.S. Abrams et al., Phys. Rev. Letters 25, 617 (1970);

C.I. Pols et al., Nucl. Phys. B25, 109 (1970);

SABRE Collaboration, Nucl. Phys. BI7, 289 (1970);

B. Werner et al., Nucl. Phys. B23, 37 (1970);

Munich-CERN-Brussels Collaboration, Munich Preprint, March (1971);

CERN-Brussels Collaboration, Nuovo Cimento $\underline{46 \mathrm{~A}}, 539$ (1966)

A.M. Boyarski et al., Phys. Rev. Letters 25, 695 (1970).

3) If non-diffractive baryon number two amplitudes are real, then

$$
\begin{aligned}
\sqrt{p n \rightarrow n p} & =\left|\sqrt{\frac{1}{2}\left(\Sigma^{-} p \rightarrow \Sigma^{o} n\right)} \pm \sqrt{\frac{3}{2}\left(\Sigma^{-} p \rightarrow \Lambda n\right)}\right| \\
\sqrt{p p \rightarrow p \Delta^{+}} & =\left|\sqrt{\Sigma^{+} p \rightarrow \Sigma^{*^{+}} p} \pm \sqrt{\Sigma^{-} p \rightarrow \Sigma^{*^{-}} p}\right| \\
& =\left|\sqrt{\frac{1}{4}\left(\Sigma^{+} p \rightarrow \Sigma^{+} \Delta^{+}\right)} \pm \sqrt{\frac{1}{2}\left(\Lambda p \rightarrow \Sigma^{-} \Delta^{++}\right)}\right|
\end{aligned}
$$

The ambiguity in sign is, of course, related to the ambiguity in the sign of the amplitude. 


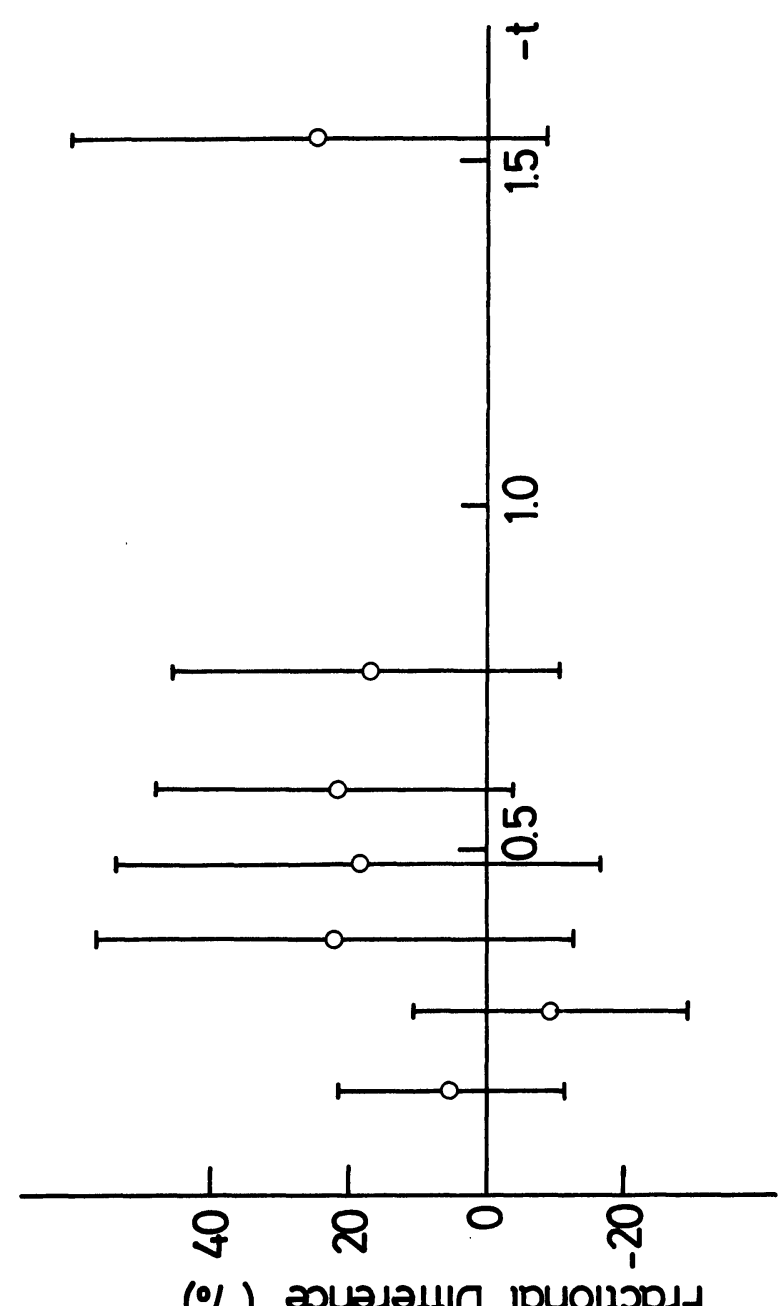

$\frac{5}{11}$

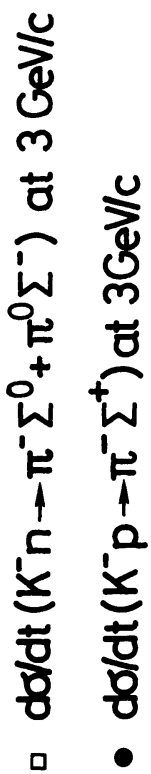

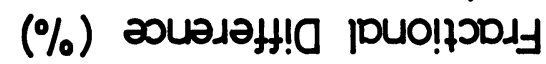

$|\perp| \perp \mid \perp$ 


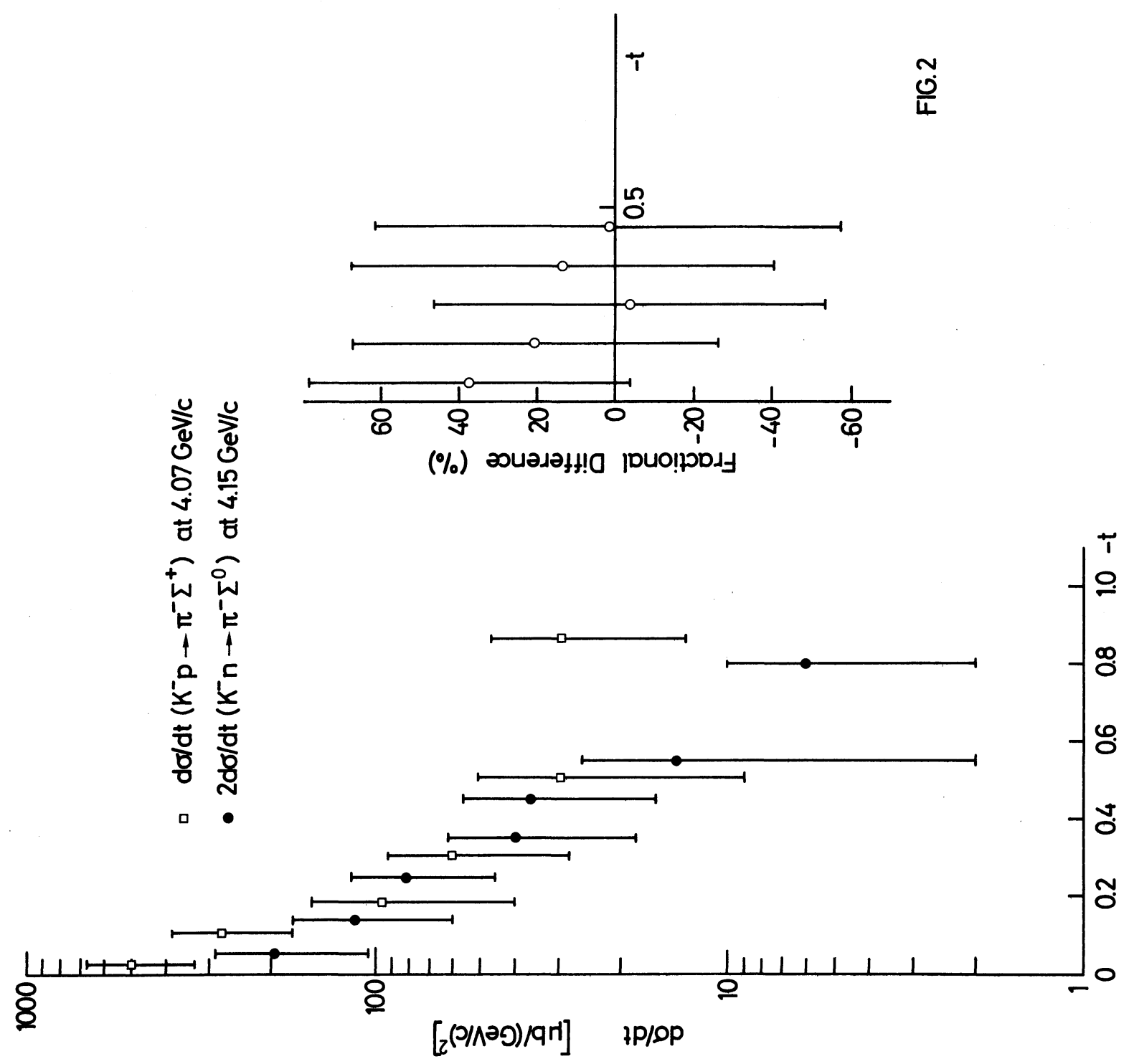



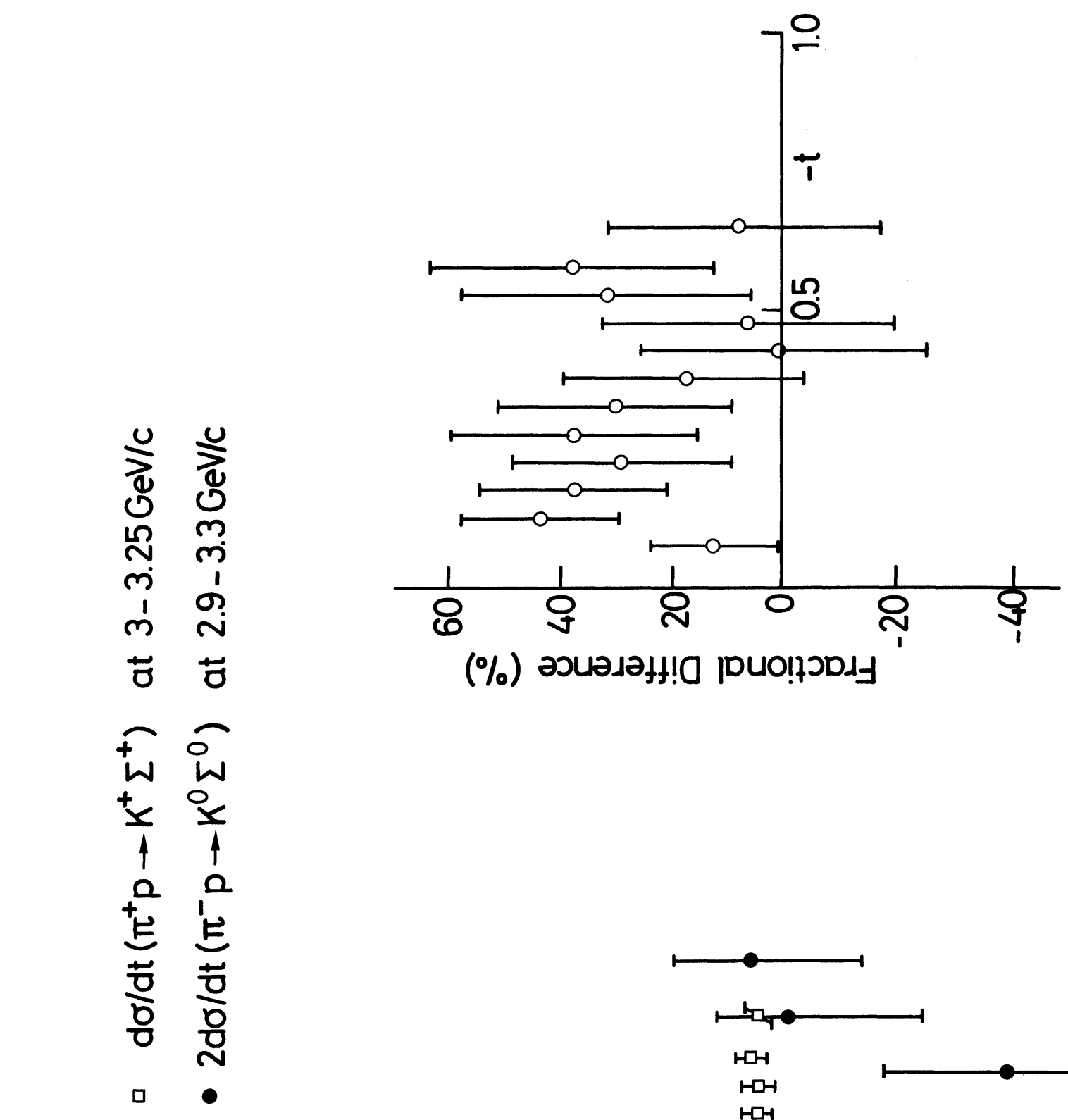

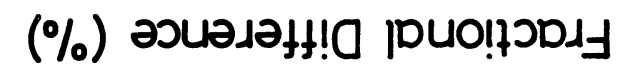

$\frac{9}{11}$
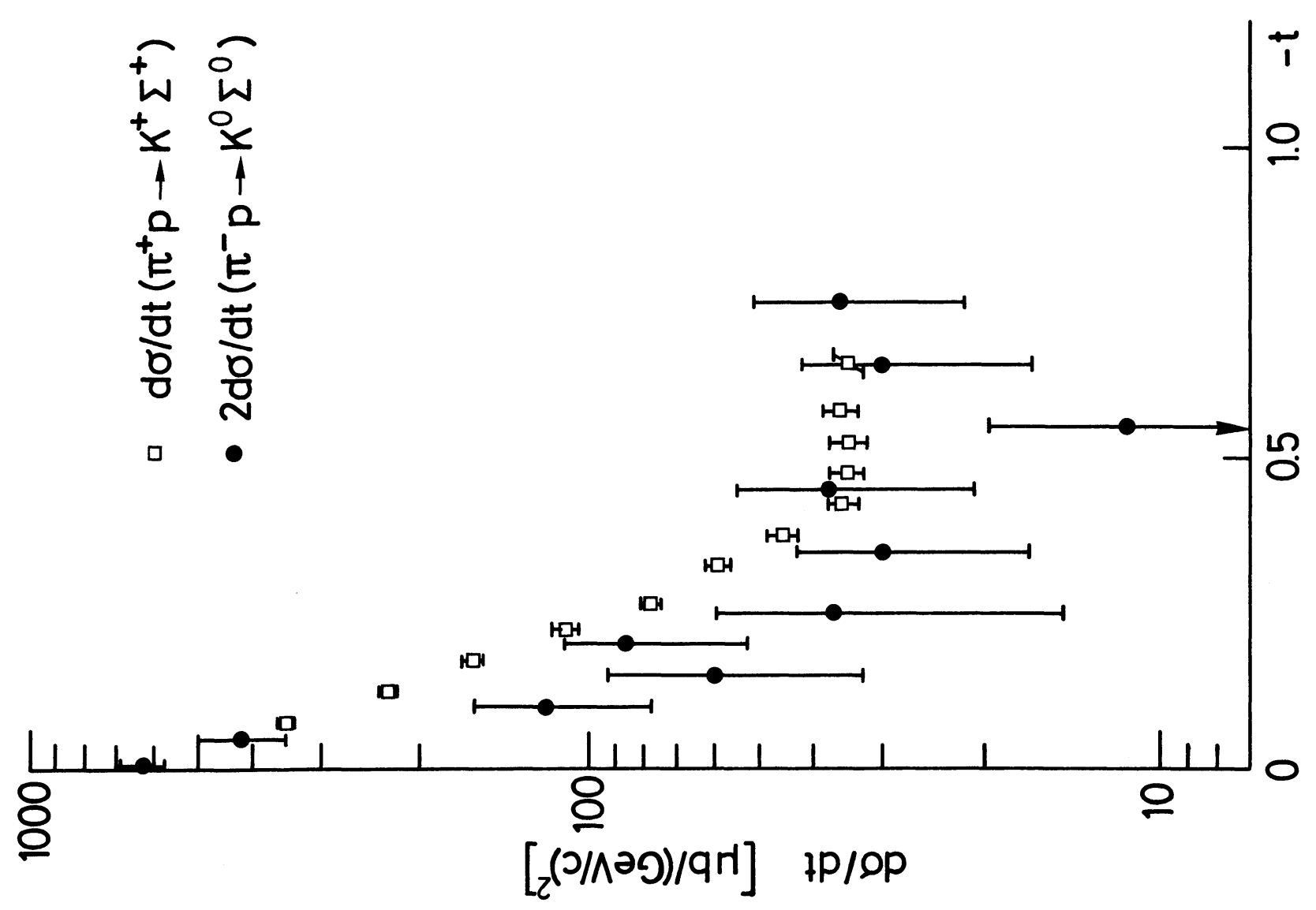

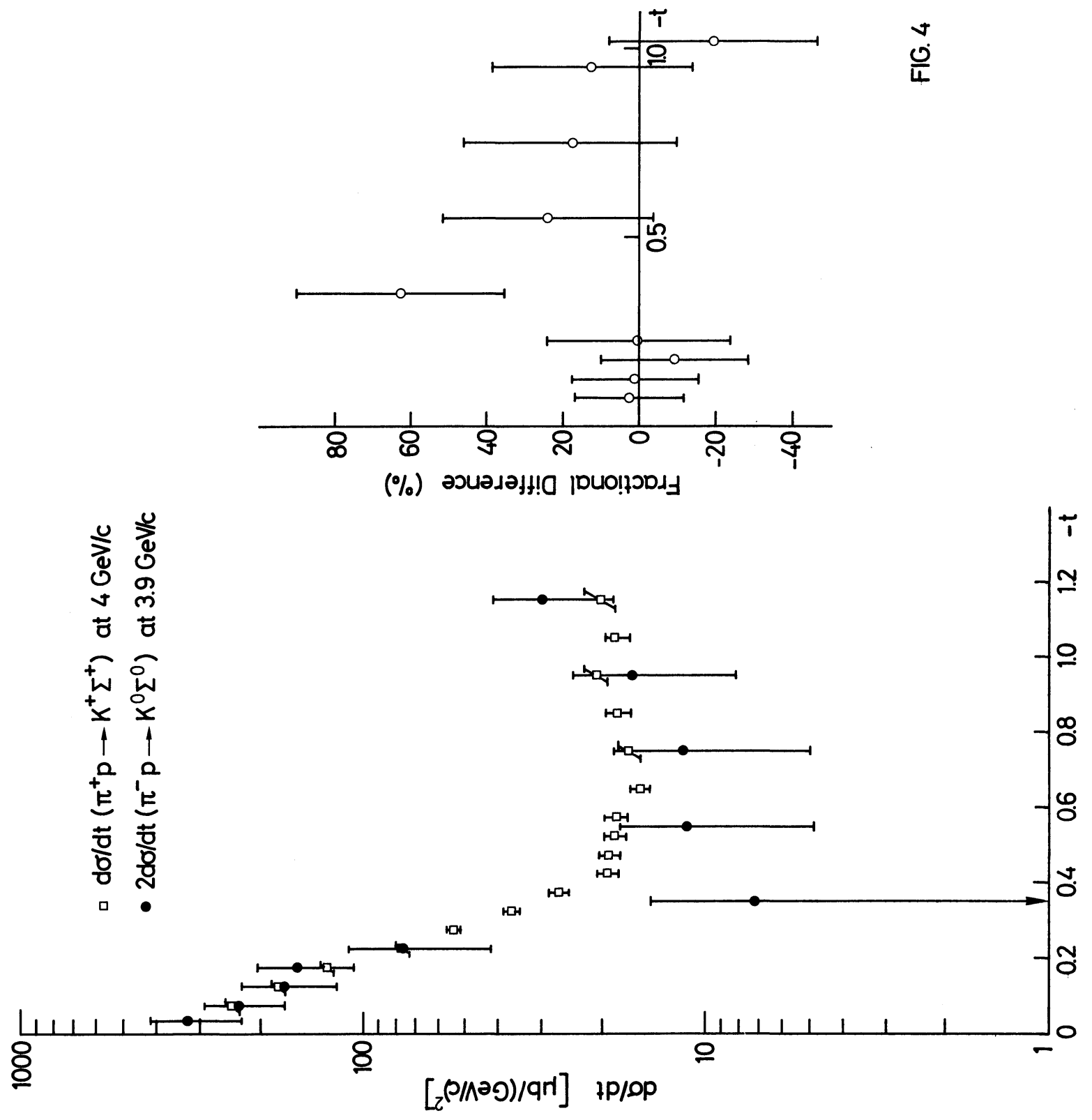


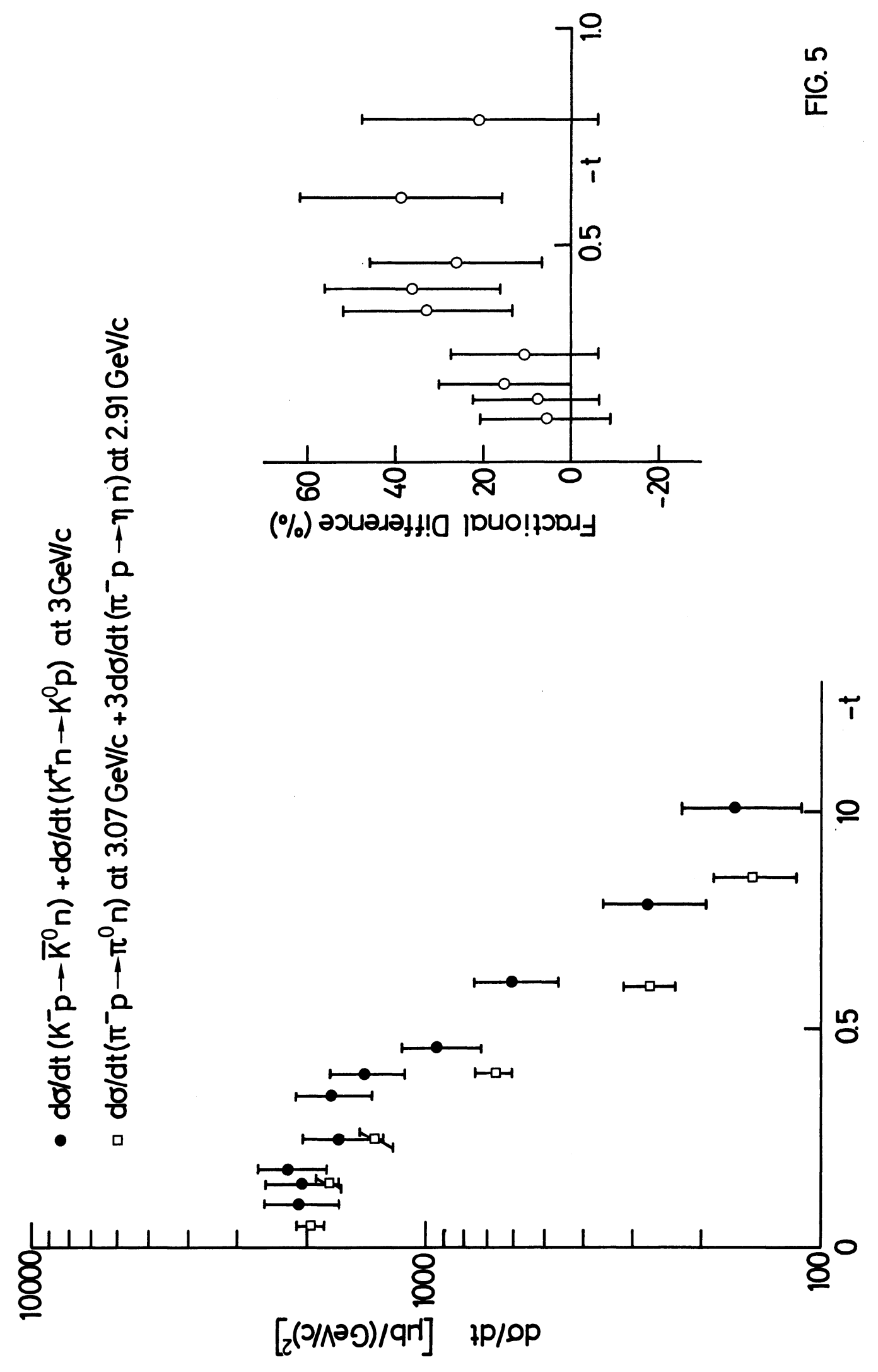



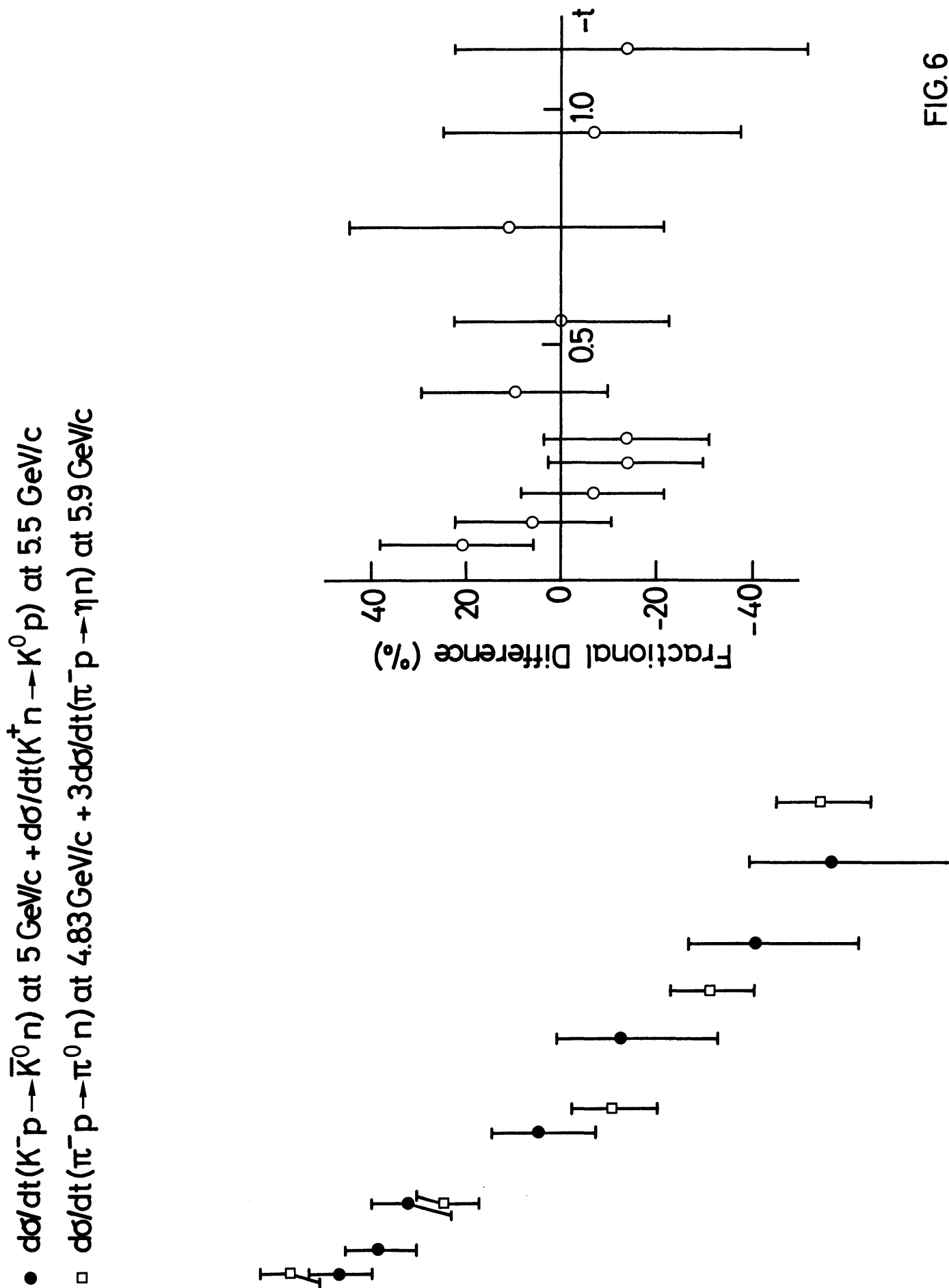

0
$\frac{0}{4}$

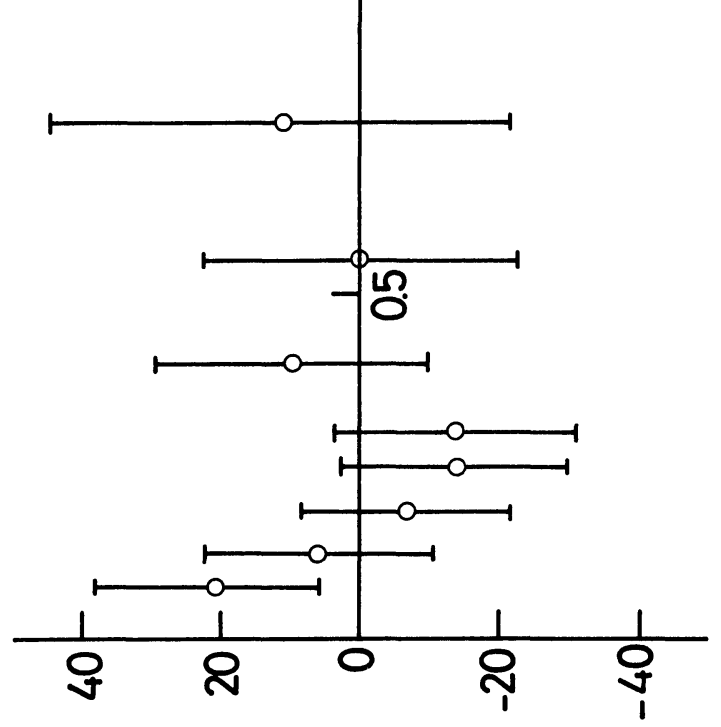

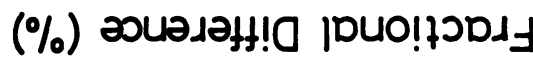
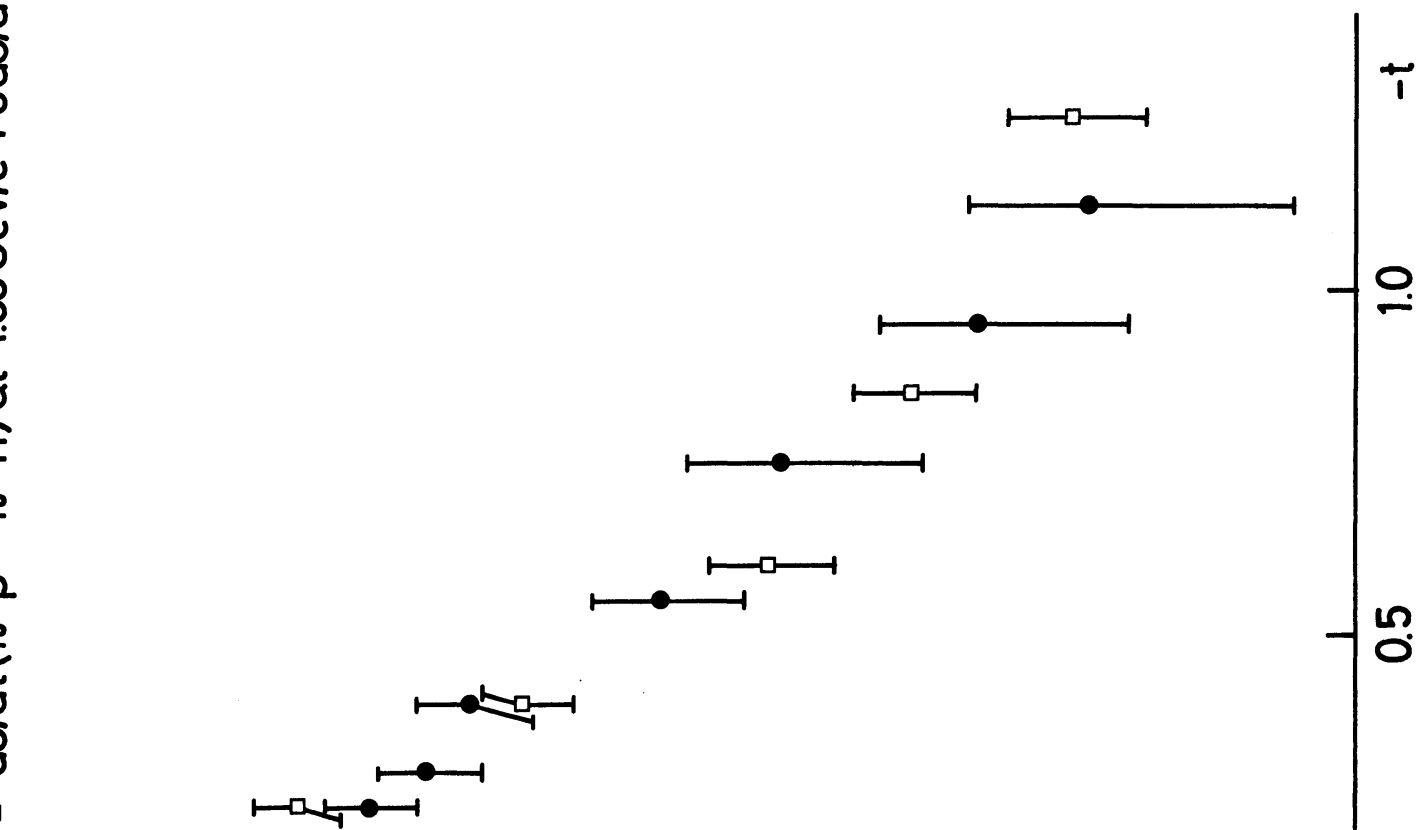


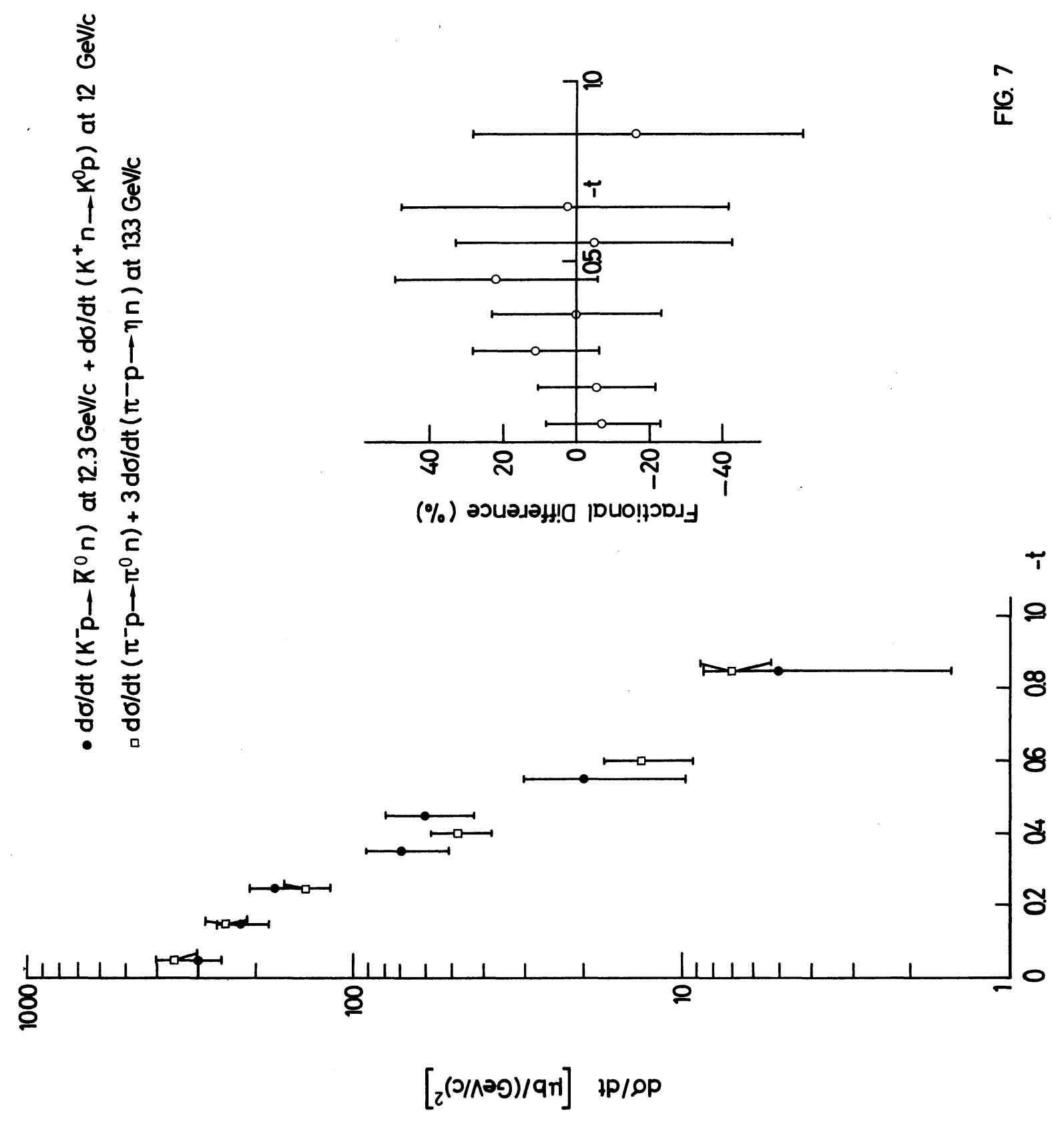




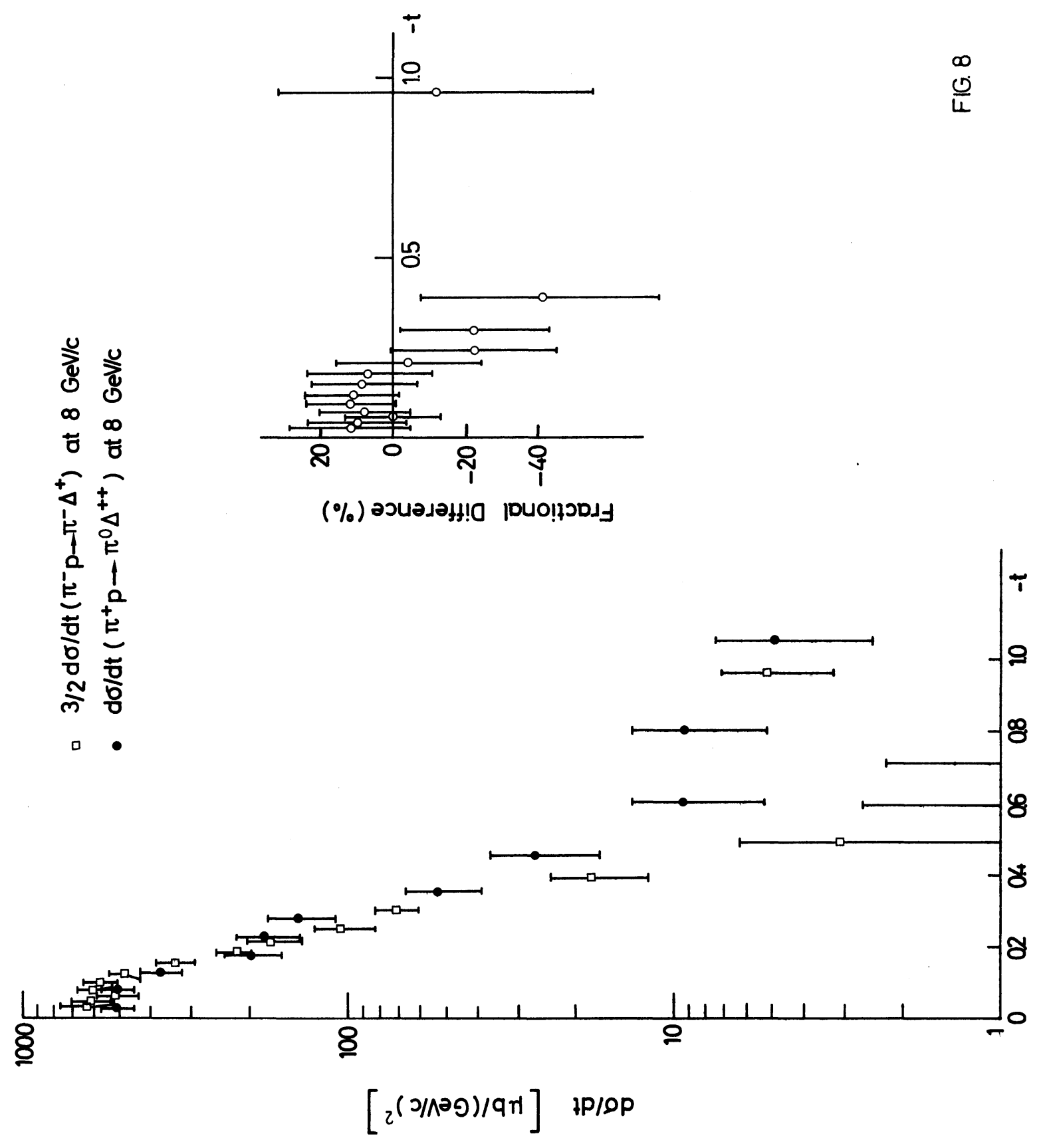




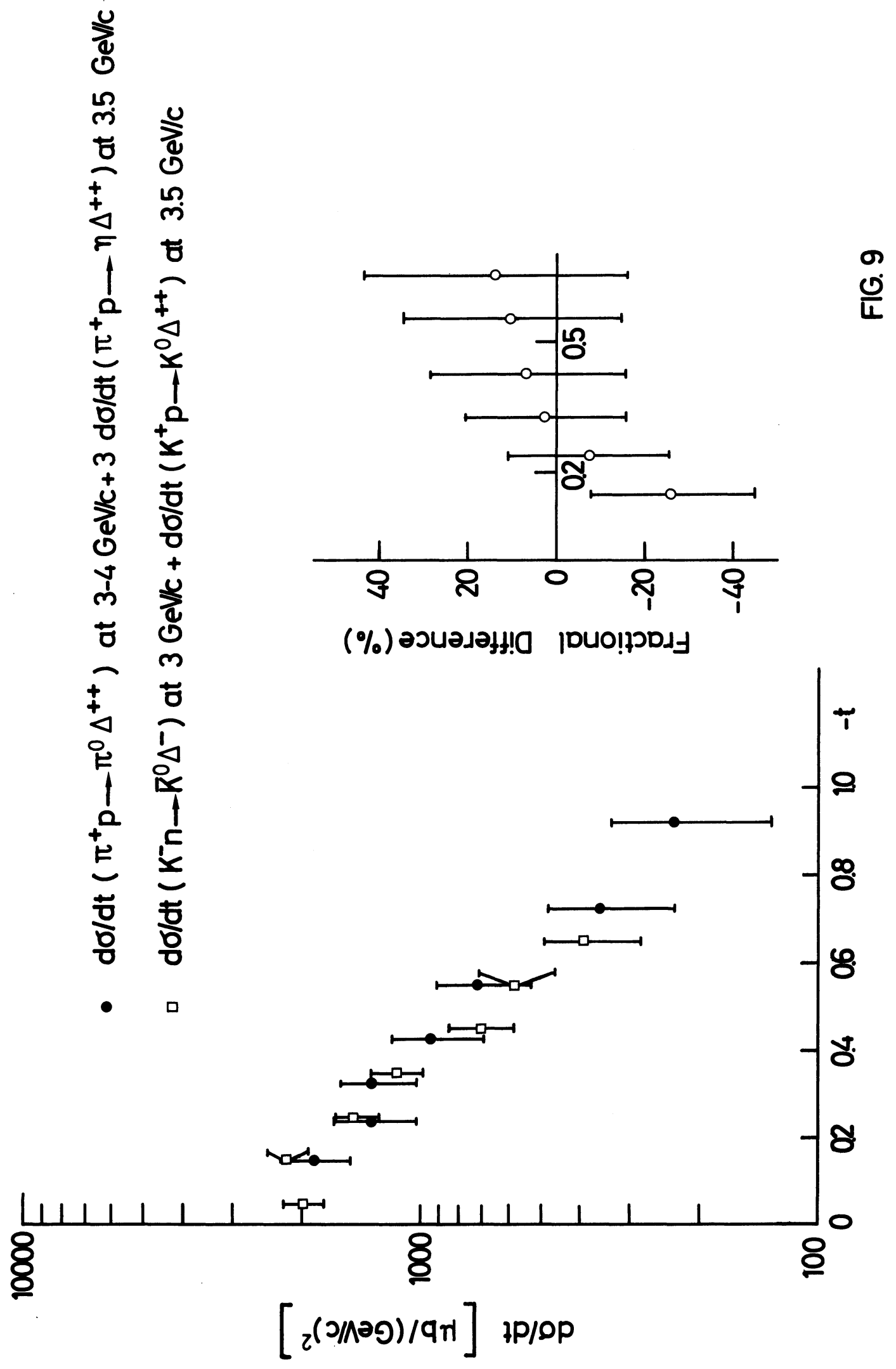




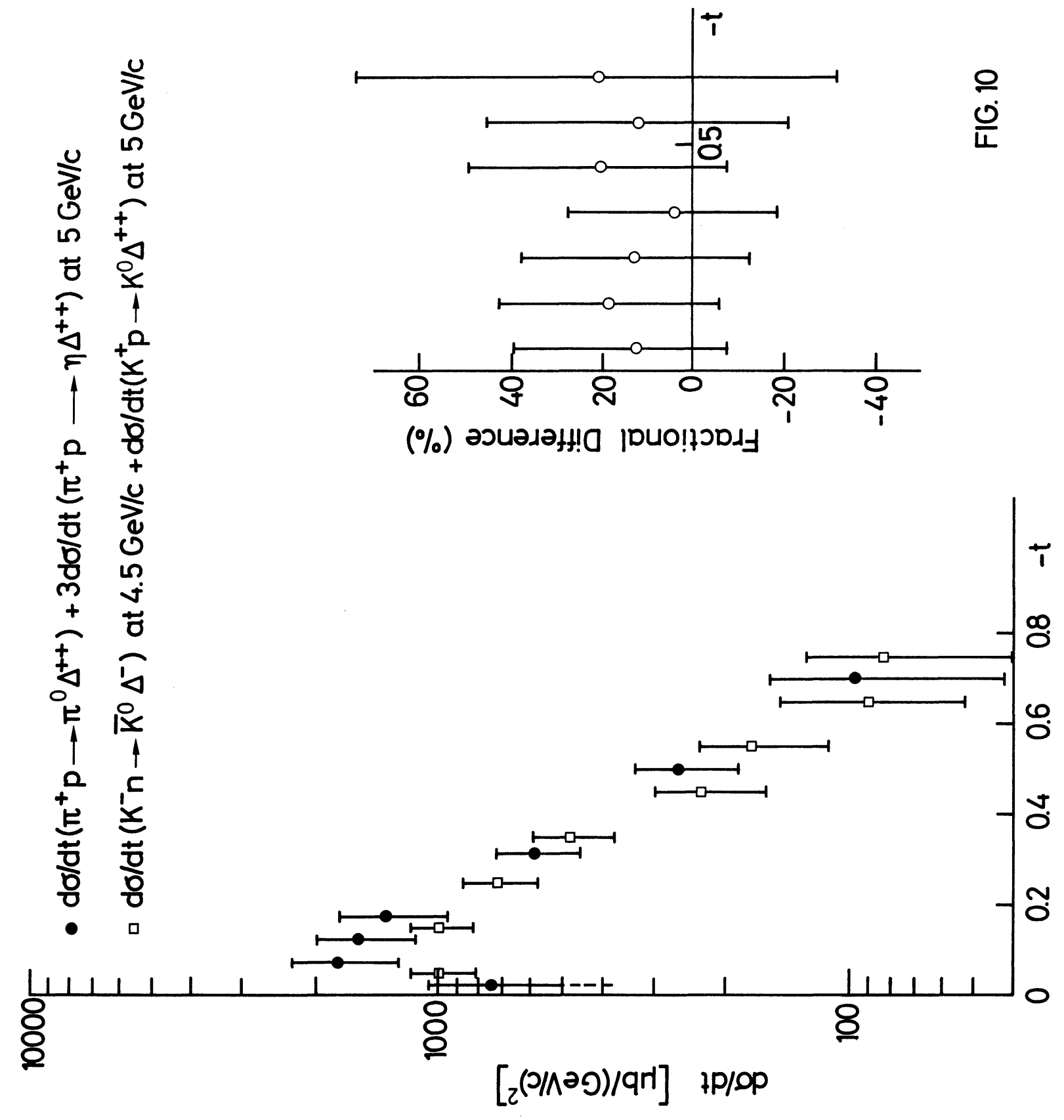




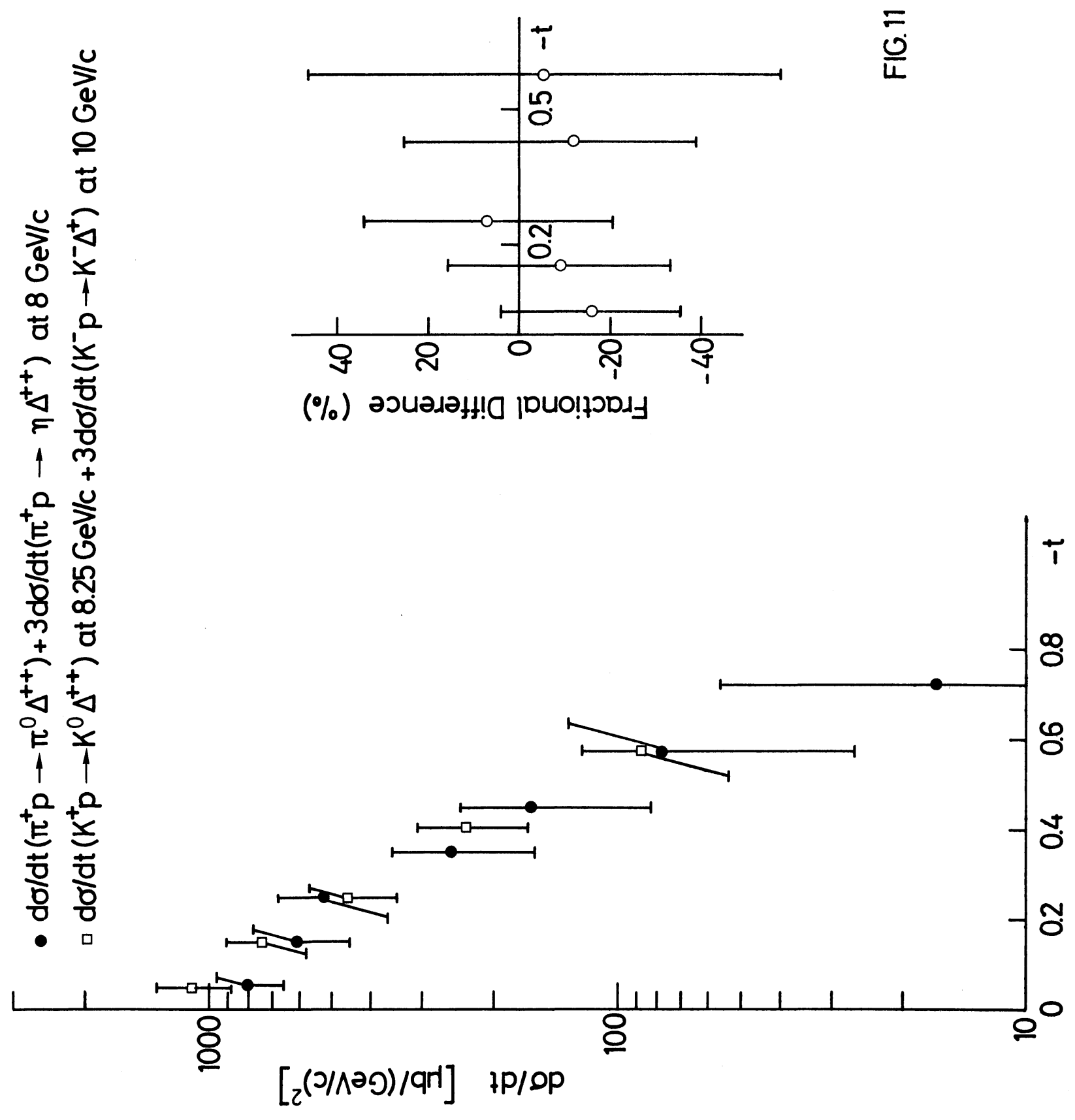




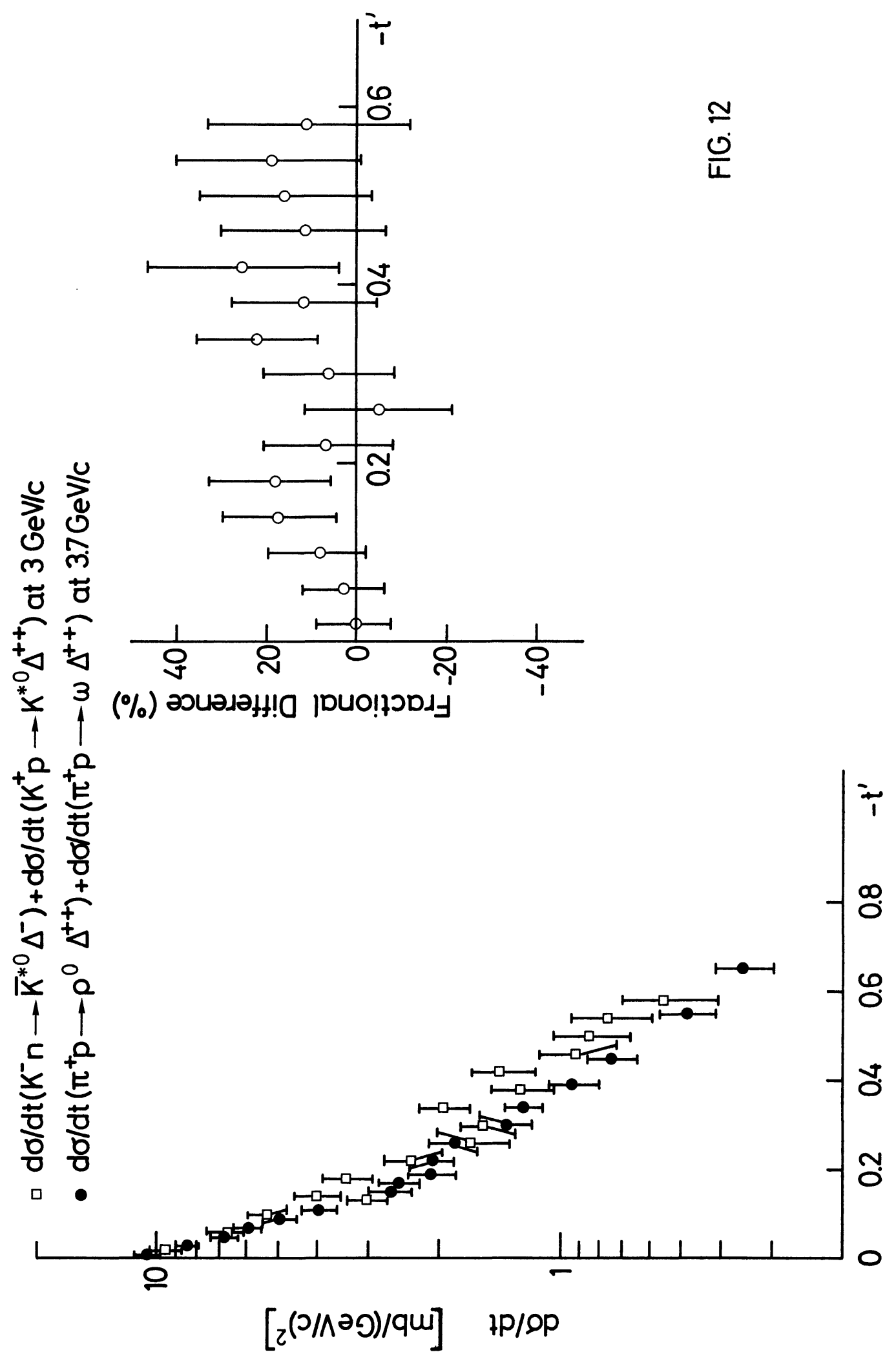




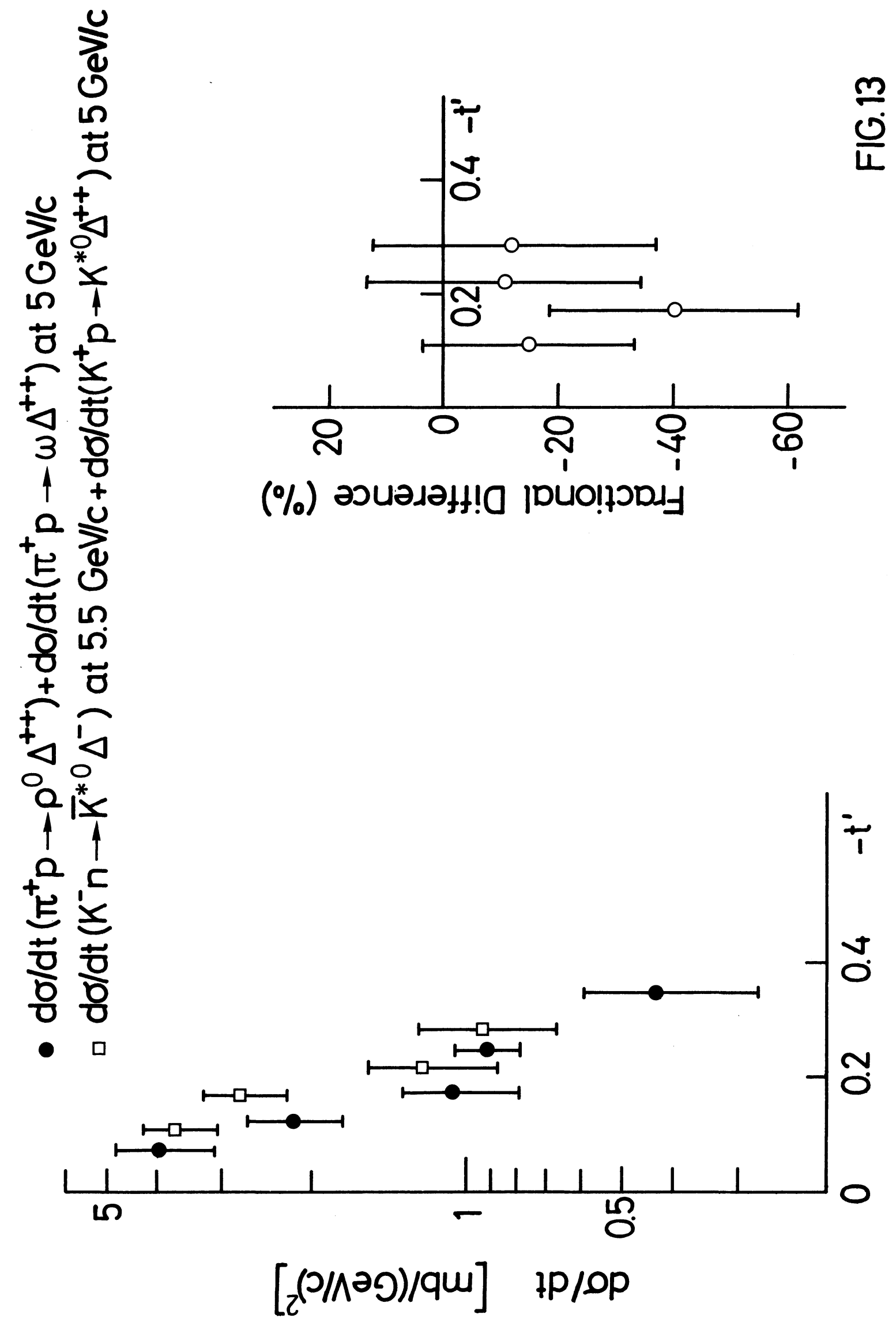




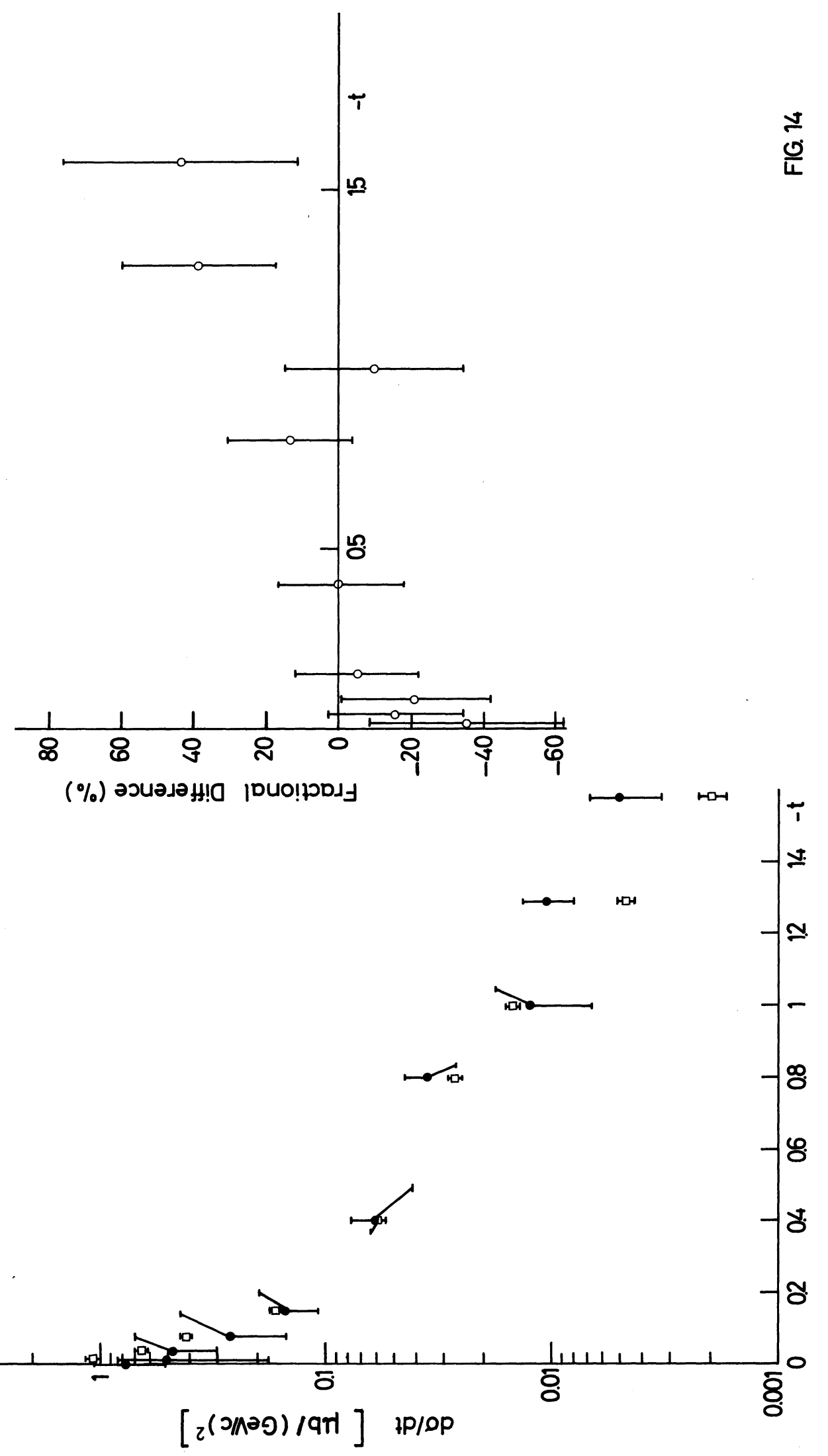




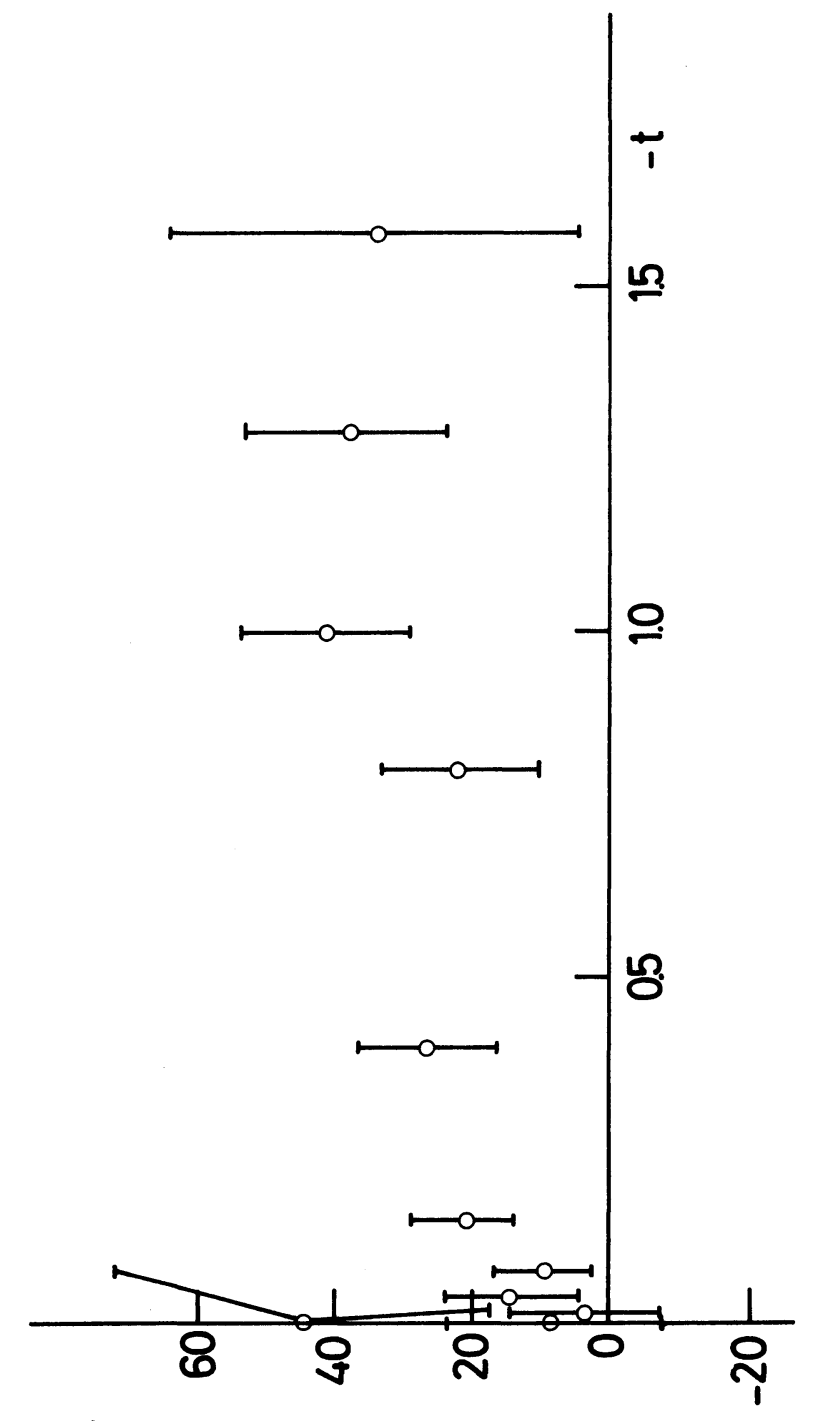

(\%) әวนวנəH!!
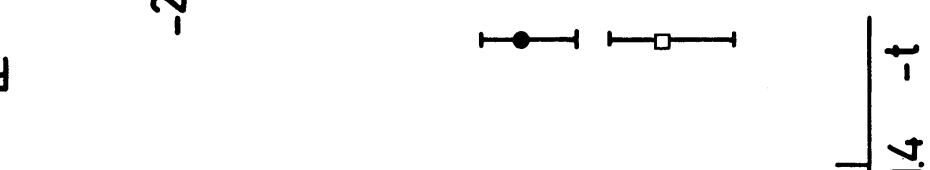

ํㅡ

ㅎ

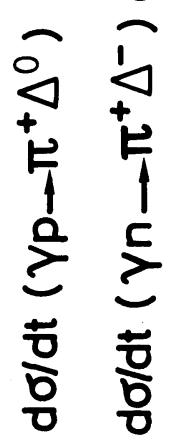

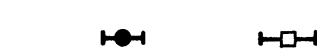

मar $\mapsto \square-1$

H HOH

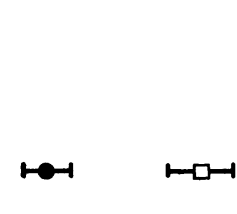

m

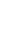

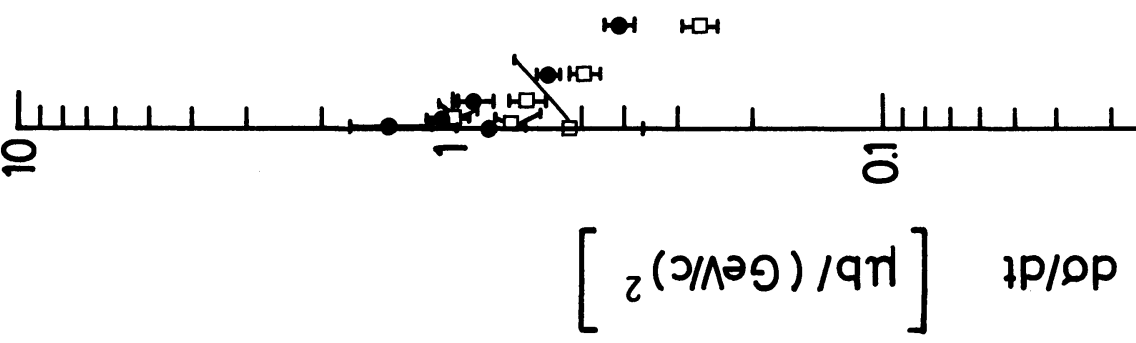

